\title{
Adaptive Retrodiction Particle PHD Filter for Multiple Human Tracking
}

\author{
Pengming Feng, Student Member, IEEE, Wenwu Wang, Senior Member, IEEE, \\ Syed Mohsen Naqvi, Senior Member, IEEE, and Jonathon Chambers, Fellow, IEEE
}

\begin{abstract}
The probability hypothesis density (PHD) filter is well known for addressing the problem of multiple human tracking for a variable number of targets, and the sequential Monte Carlo (SMC) implementation of the PHD filter, known as the particle PHD filter, can give state estimates with nonlinear and non-Gaussian models. Recently, Mahler et al. have introduced a PHD smoother to gain more accurate estimates for both target states and number. However, as highlighted by Psiaki in the context of a backward-smoothing extended Kalman filter, with a non-linear state evolution model the approximation error in the backward filtering requires careful consideration. Psiaki suggests to minimise the aggregated least-squares error over a batch of data. We instead use the term retrodiction PHD (Retro-PHD) filter to describe the backward filtering algorithm in recognition of the approximation error proposed in the original PHD smoother, and we propose an adaptive recursion step to improve the approximation accuracy. This step combines forward and backward processing through the measurement set and thereby mitigates the problems with the original PHD smoother when the target number changes significantly and the targets appear and disappear randomly. Simulation results show the improved performance of the proposed algorithm and its capability in handling a variable number of targets.
\end{abstract}

Index Terms-adaptive filter, PHD filter, Retro-PHD, multiple human tracking.

\section{INTRODUCTION}

$\mathbf{V}$ IDEO signal processing based human tracking is becoming increasing popular because of its wide potential applications. However, tracking multiple human targets has many challenges such as variable number of targets, targets occlusion and target states estimation. To solve these challenges, especially the variable number of targets, one way is to find explicit associations between measurements and targets, and then to filter the measurement to individual targets[1]. However, due to the additional processing required for the estimation and data association for each individual target, the computational complexity of these methods grows exponentially with the increase in the number of targets [2]. Based upon the concept of a Random Finite Set (RFS) [3],

This work was supported by the Engineering and Physical Sciences Research Council (EPSRC) Grant number EP/K014307 and the MOD University Defence Research Collaboration in Signal Processing.

P. Feng, S. M. Naqvi and J. A. Chambers are with the Communications, Sensors, Signal and Information Processing Group, School of Electrical and Electronic Engineering, Newcastle University, United Kingdom, e-mails: \{P.Feng2, Mohsen.Naqvi, Jonathon.Chambers\}@ newcastle.ac.uk.

W. Wang is with the Center for Vision Speech and Signal Processing, Department of Electrical and Electronic Engineering, University of Surrey, United Kingdom, e-mail: w.wang@surrey.ac.uk.

Copyright (c)2016 IEEE. the PHD filter [4] is proposed to handle the varying target cardinality and to reduce the computational complexity by only employing the first-order moment of the posterior density [5]. The PHD is the intensity function of a finite point process and, in practice, both the number of targets and their states are extracted from the PHD surface, which is a spatial distribution of target number [5] [6]. However, as mentioned in [7], the PHD filter depends on the current measurement set, so in the case of a low number of observable measurements, its performance is limited.

In order to achieve more observable measurements, a forward-backward filtering algorithm has been utilized for the particle PHD filter [8][9], which provides backward estimation from the aid of delayed measurement set. Improved performance has been shown in [8] and [9] by employing the PHD smoothing algorithm in multiple target tracking. However, in [10], Psiaki has pointed out that, when using the smoothing algorithm for a nonlinear filtering system, it is necessary to consider a batch of data and minimize the aggregated error over the batch at each time step to mitigate the approximation error. As a consequence, when attempts backward processing with embedded approximations (such as in PHD smoothing, with a nonlinear state model) the use of the term smoother should be avoided. So in this letter, we adopt the term retrodiction to represent the backward filtering process in [9]. When evaluating the Retro-PHD algorithm, we found although the approach can improve the tracking results over the PHD filter; its performance deteriorates with an increasing number of targets appearing and disappearing in the monitored area. In such a scenario, more false measurements for backward estimation are introduced by false alarm or missed detection.

Adaptive filters [11][12] on the other hand are a widely used signal processing technique for their exploitation of recursion in tracking [11]. Following the idea of combination of adaptive filters proposed in [11], in this letter, a new method for the Retro-PHD filter is proposed by using an adaptive recursion step, in which the measurements from both forward and backward processing are employed for target state estimation. Measurements are utilized to calculate the adaptive weights, which are then used to enhance the tracking results. Simulations using sequences from the CAVIAR [13] and the PETS2009 [14] datasets will show that the proposed adaptive Retro-PHD outperforms the state-of-art particle PHD filter and the original Retro-PHD filter [9]. Other recent multiple human target trackers such as cardinality PHD filter [15] and multiBernoulli filter [16] are not included in this short study as they do not involve backward/retrodiction processing. 


\section{SMC PHD FILTERING AND RETRO-PHD FILTERING}

\section{A. Particle PHD filtering}

In this work, the particle PHD filter is employed for tracking because it performs well in the scenarios of non-Gaussian noise and non-linear models [17]. We assume the set of targets $\left\{\mathbf{x}_{k}^{m}\right\}_{m=1}^{m=M_{k}}$ includes the states of all the human targets, where $M_{k}$ is the number of targets at time $k$ and $m$ is the target index. Denote the measurement set at time $k$ as matrix $\mathbf{Z}_{k}$, which includes vector $\mathbf{z}_{k}$ as each individual measurement. The basic principle of importance sampling in the particle filter is to represent a PDF $p\left(\mathbf{X}_{k} \mid \mathbf{Z}_{k}\right)$ by a set of random particles $\mathbf{x}_{k}^{i}$ having associated weights $w_{k}^{i}$, where $\mathbf{X}_{k}=$ $\left\{\mathbf{x}_{k}^{i}, i=1, \ldots, N\right\}$, which denotes all $N$ particles utilized to describe the states of all human targets at time $k$ [18], and for each particle, $\mathbf{x}_{k}^{i}=\left[p_{k, x}^{i}, p_{k, y}^{i}, v_{k, x}^{i}, v_{k, y}^{i}, h_{k}^{i}, w_{k}^{i}\right]^{T} \in \mathbb{R}^{6}$ denotes the state of the particle at discrete time $k$, including the 2D position $\left(p_{k, x}^{i}, p_{k, y}^{i}\right)$, velocity $\left(v_{k, x}^{i}, v_{k, y}^{i}\right)$, height and width of targets $h_{k}^{i}, w_{k}^{i}$; where $(\cdot)^{T}$ denotes the transpose operator and subscripts $x, y$ are the horizontal and vertical coordinates of the target. Denoting $D(\cdot)$ as the PHD at discrete time associated with the multi-target posterior density, the prediction and updating step for the particle PHD filter can be described as follows [17]:

1. Prediction: Drawing survived particles $\tilde{\mathbf{x}}_{k}^{i}$ from the predicted particle set and feeding into the prediction model of the particle PHD filter, which is described as [19]

$$
D\left(\mathbf{X}_{k} \mid \mathbf{Z}_{k-1}\right)=\int \phi\left(\tilde{\mathbf{x}}_{k}^{i} \mid \mathbf{X}_{k}\right) D\left(\mathbf{X}_{k-1} \mid \mathbf{Z}_{k-1}\right) \delta \tilde{\mathbf{x}}_{k}^{i}+\Upsilon_{k}\left(\mathbf{X}_{k}\right)
$$

where $\Upsilon_{k}(\cdot)$ is the intensity function of the new target birth RFS, $\phi(\cdot)$ is the analogue of the state transition probability in the single target case which is calculated as

$$
\phi\left(\tilde{\mathbf{x}}_{k}^{i} \mid \mathbf{X}_{k}\right)=e\left(\tilde{\mathbf{x}}_{k}^{i} \mid \mathbf{X}_{k}\right)+\beta\left(\tilde{\mathbf{x}}_{k}^{i} \mid \mathbf{X}_{k}\right)
$$

for which $e(\cdot)$ is the probability that the targets still exist at time $k$ and $\beta(\cdot)$ is the intensity of the RFS for spawned targets. When exploiting the PHD filter with the particle filter, the PHD of the states is represented by the weights of the particles, which include the survived particles and new-born particles. In the traditional particle PHD filter, the particles employed to describe the new-born targets are selected randomly in the video scene, however, to make more accurate prediction, the new-born targets can be obtained by employing a background subtraction step, which is described in [20] in detail. In this case, assuming at time $k, J_{k}$ new-born particles are obtained from the background subtraction, the initial weights employed to represent the new-born targets are given as

$$
\tilde{w}_{k \mid k-1}^{i}=\frac{1}{J_{k}}
$$

then the weights are fed into (1) and to obtain the particle set for the particle PHD prediction.

$$
\left\{\tilde{\mathbf{x}}_{k}^{i}, \tilde{w}_{k \mid k-1}^{i}\right\}_{i=1}^{i=N+J_{k}}
$$

where $i$ is the index of all particles. The predicted weights from the particle PHD filter prediction step are given as

$$
\tilde{w}_{k \mid k-1}^{i}=\left\{\begin{array}{l}
\phi\left(\tilde{\mathbf{x}}_{k}^{i} \mid \mathbf{X}_{k}\right) w_{k-1}^{i} \quad i=1, \ldots, N \\
\frac{\Upsilon_{k}}{J_{k}} \quad i=N+1, \ldots, N+J_{k}
\end{array}\right.
$$

2. Update: In the application of the particle PHD filter, the PHD $D(\cdot)$ is represented by the weights of the particles, once the new set of observations is available, we can substitute the approximation of $D\left(\mathbf{X}_{k} \mid \mathbf{Z}_{k-1}\right)$ and the weights of each particle are updated based upon the receipt of the measurements $\mathbf{Z}_{k}$ as [19]

$$
\tilde{w}_{k}^{i}=\left[p_{M}\left(\tilde{\mathbf{x}}_{k}^{i}\right)+\sum_{\forall \mathbf{z}_{k} \in \mathbf{Z}_{k}} \frac{\psi_{k, \mathbf{z}_{k}}\left(\tilde{\mathbf{x}}_{k}^{i}\right)}{\kappa_{k}+C_{k}\left(\mathbf{z}_{k}\right)}\right] \tilde{w}_{k \mid k-1}^{i}
$$

where

$$
C_{k}\left(\mathbf{z}_{k}\right)=\sum_{i=1}^{N+J_{k}} \psi_{k, \mathbf{z}_{k}}\left(\tilde{\mathbf{x}}_{k}^{i}\right) \tilde{w}_{k \mid k-1}^{i}
$$

and $\psi_{k, \mathbf{z}_{k}}\left(\tilde{\mathbf{x}}_{k}^{i}\right)=\left(1-p_{M}\left(\tilde{\mathbf{x}}_{k}^{i}\right)\right) g\left(\mathbf{z}_{k} \mid \tilde{\mathbf{x}}_{k}^{i}\right)$ is the single target likelihood. In this work, the likelihood of each particle is calculated by histograms of colour and oriented gradient features of human targets [21], by assuming that the noise on the colour and oriented gradient likelihood function is Gaussian

$$
g\left(\mathbf{z}_{k} \mid \tilde{\mathbf{x}}_{k}^{i}\right) \sim \mathbb{N}\left(\mathbf{z}_{k} ; 0, \sigma_{g}^{2}\right)=\frac{1}{\sqrt{2 \pi \sigma_{g}^{2}}} \exp \left(-\frac{\left\{G\left(\mathbf{x}_{k}^{i}\right)\right\}^{2}}{2 \sigma_{g}^{2}}\right)
$$

where $\sigma_{g}^{2}$ is the variance of the noise for the colour and gradient likelihood and $G\left(\tilde{\mathbf{x}}_{k}^{i}\right)$ is the colour similarities calculated as the Bhattacharyya distance between the reference measurement and the histogram of colour and oriented gradient $\mathbf{s}(\cdot)$ extracted from the rectangle area centered around the particle location, which can be calculated as

$$
G\left(\tilde{\mathbf{x}}_{k}^{i}\right)=\sqrt{1-\mathbf{s}\left(\tilde{\mathbf{x}}_{k}^{i}\right)^{T} \mathbf{z}_{k}}
$$

After the updating step of the particle PHD filter, the number of targets is calculated by the sum of all the weights for particles as [19]

$$
\tilde{M}_{k}=\operatorname{int}\left(\sum_{i=1}^{N+J_{k}} \tilde{w}_{k}^{i}\right)
$$

where $\operatorname{int}(\cdot)$ takes the nearest integer. After calculating the number of targets, a resampling step is employed as described in [2] in order to limit the number of particles, thereby avoiding the number of particles growing exponentially. Then the tracking results $\left\{\tilde{\mathbf{x}}_{k}^{m}\right\}_{m=1}^{m=\tilde{M}_{k}}$ are obtained from the particle PHD filter, where $(\tilde{*})$ denotes the states from the PHD filtering algorithm.

\section{B. Particle Retro-PHD Filtering}

The Retro-PHD filter is employed to use more measurements beyond the current time by processing information from later stages in an approximate manner, and can achieve more accurate tracking results. Similar to forward particle PHD filtering, the retrodiction step is also generalized by the RFS [9] [8]. The algorithm is concerned with the density at time $t=k-L$, where $L$ is the time lag. When employing the particle Retro-PHD filter, the retrodicted particle weights at time $t$ are evaluated using the backward iterations using filter outputs $\left\{\tilde{\mathbf{x}}_{k}^{i}, \tilde{w}_{k}^{i}\right\}_{i=1}^{i=N}$ for $t=k-L, \ldots, k$. The particle weights 
from the backward filtering stage are computed as derived in [8]:

$$
\hat{w}_{t \mid k}^{i}=\tilde{w}_{t}^{i}\left[e\left(\tilde{\mathbf{x}}_{t}^{i}\right) \sum_{q=1}^{N} \frac{\tilde{w}_{t+1 \mid k}^{q} f_{t+1 \mid t}\left(\tilde{\mathbf{x}}_{t+1}^{q} \mid \tilde{\mathbf{x}}_{t}^{i}\right)}{\mu_{t+1 \mid t}^{q}}+\left(1-e\left(\tilde{\mathbf{x}}_{t}^{i}\right)\right)\right]
$$

where

$$
\mu_{t+1 \mid t}^{q}=\Upsilon_{t+1}\left(\tilde{\mathbf{x}}_{t+1}^{q}\right)+\sum_{r=1}^{N} \tilde{w}_{t}^{r} \times\left\{e\left(\tilde{\mathbf{x}}_{t+1}^{r}\right) f_{t+1 \mid t}\left(\tilde{\mathbf{x}}_{t+1}^{q} \mid \tilde{\mathbf{x}}_{t}^{r}\right)\right\}
$$

and the conversion function is given as:

$$
f_{t \mid t-1}\left(\tilde{\mathbf{x}}_{t}^{i} \mid \tilde{\mathbf{x}}_{t-1}^{i}\right)=\frac{\exp \left(-\frac{\left(\tilde{\mathbf{x}}_{t}^{i}-\mathbf{F}\left(\tilde{\mathbf{x}}_{t-1}^{i}\right)\right)^{T}\left(\tilde{\mathbf{x}}_{t}^{i}-\mathbf{F}\left(\tilde{\mathbf{x}}_{t-1}^{i}\right)\right)}{2 \sigma_{f}^{2}}\right)}{\sqrt{\operatorname{det}\left(2 \pi \sigma_{f}^{2}\right)}}
$$

where $\sigma_{f}^{2}$ is the variance of the conversion function and $\mathbf{F}(\cdot)$ is the state transformation matrix. After obtaining the particle set $\left\{\hat{\mathbf{x}}_{t}^{i}, \hat{w}_{t}^{i}\right\}_{i=1}^{i=N}$ from the particle Retro-PHD filter, the number and states of the human targets are obtained in the same way as described in Section II-A and in order to mitigate the effects of particle depletion, a resampling step is employed as described in [9]. The tracking results from the particle Retro-PHD filter are represented as $\left\{\hat{\mathbf{x}}_{k}^{m}\right\}_{m=1}^{m=\hat{M}_{k}}$.

\section{AdAptive Solution for PARTicle Retro-PHD FILTER}

From the above steps, results from both the forward and backward filtering process are obtained, in which the forward measurements are utilized in the filtering algorithm and backward measurements are utilized in the retrodiction algorithm to estimate the target states, which are represented with the black lines and the blue lines in the graphical representation in Fig. 1 respectively. However, as mentioned in Section 1 .

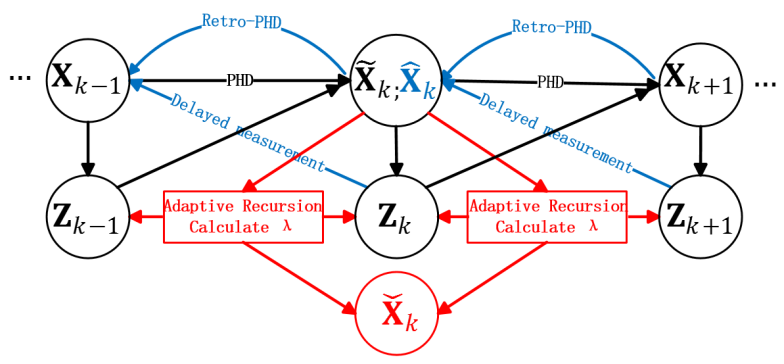

Fig. 1. Graphical comparison between PHD filtering, Retro-PHD filtering and the proposed adaptive Retro-PHD filtering algorithms, where the black lines denote the PHD filtering algorithm, the blue lines denote the Retro-PHD filtering algorithm and the red lines denote the proposed adaptive retrodiction step.

the accuracy of the backward state estimation from the RetroPHD filter is limited because of the limitation of the accuracy of the delayed measurements. When the number of targets changes and the environmental noise increases, the delayed measurements are easily influenced by missed detection and false alarms, which will cause false measurements, and hence reduce the accuracy of the Retro-PHD filter. To address this issue, an adaptive step is designed for combining the forward and retrodiction state estimation. As shown in the red lines of Fig. 1], an adaptive scalar parameter $\lambda$ is employed to weight the results given from the forward filtering and retrodiction filtering algorithms, which is calculated by the similarity of the measurement set over discrete time samples. Assuming the measurement set at times $k-1, k, k+1$ are $\mathbf{Z}_{k-1}, \mathbf{Z}_{k}$ and $\mathbf{Z}_{k+1}$ respectively, which are generated by the target states being tracked, and the measurement RFS is extracted from the RFS of the tracking results, which includes $M_{k}$ targets. Adaptive parameter $\lambda_{o}, o \in\{$ filtering, retrodiction $\}$ are calculated as:

$$
\begin{aligned}
\lambda_{\text {filtering }} & =\frac{\sum_{i=1}^{M_{k}} \sum_{r=1}^{M_{k-1}} \exp \left(-\frac{\left(\mathbf{z}_{k}^{i}-\mathbf{z}_{k-1}^{r}\right)^{T}\left(\mathbf{z}_{k}^{i}-\mathbf{z}_{k-1}^{r}\right)}{2 \sigma_{\lambda}^{2}}\right)}{M_{k-1}} \\
\lambda_{\text {retrodiction }} & =\frac{\sum_{i=1}^{M_{k}} \sum_{r=1}^{M_{k+1}} \exp \left(-\frac{\left(\mathbf{z}_{k}^{i}-\mathbf{z}_{k+1}^{r}\right)^{T}\left(\mathbf{z}_{k}^{i}-\mathbf{z}_{k+1}^{r}\right)}{2 \sigma_{\lambda}^{2}}\right)}{M_{k+1}}
\end{aligned}
$$

and by normalizing $\lambda_{\text {filtering }}$ and $\lambda_{\text {retrodiction }}$, the weight value for the forward and backward measurements is given as:

$$
\lambda=\frac{\lambda_{\text {filtering }}}{\lambda_{\text {filtering }}+\lambda_{\text {retrodiction }}} .
$$

which gives convex weights to the results from both tracking and filtering. Thus the tracking position from the adaptive step is found by using a convex combination of results from both filtering and retrodiction as:

$$
\check{\mathbf{x}}_{k}^{m}=\left\{\begin{array}{l}
\tilde{\mathbf{x}}_{k}^{m} \text { if target } m \text { disappears at } k+1 \\
\lambda \tilde{\mathbf{x}}_{k}^{m}+(1-\lambda) \hat{\mathbf{x}}_{k}^{m} \text { otherwise }
\end{array}\right.
$$

where $(\check{\bullet})$ denotes the results from the adaptive recursion retrodiction step. We use this convex combination because it is a simple and and intuitive way for fusing the information and provides flexibility to automatically control the contribution of the forward and backward information adaptively. In this way, the filtering results are employed to make corrections for the results from the Retro-PHD filter based on the similarity between forward and delayed measurements, which reduces the probability of the false measurements caused by missed detection and false alarms.

In summary, for time $k>1$, the adaptive particle RetroPHD filter can be described as Algorithm 1.

\section{Simulation}

In order to evaluate the performance of the proposed adaptive particle Retro-PHD filter, sequences from the CAVIAR and PETS2009 datasets are employed, where in the CAVIAR dataset, 3-5 human targets are walking in a shopping mall environment and in the PETS2009 dataset, 3-6 human targets are walking in a campus environment, and these include human target occlusion, appearing and disappearing randomly in the scene. In this work, 1000 particles are employed to represent targets in the CAVIAR dataset and 1500 particles are employed in the PETS2009 dataset; 200 particles are employed for new-born targets in each frame. Following previous experience, as in [20], the zero-mean noise vector $\mathbf{w}_{k}$ for prediction in the state model has covariance structure 


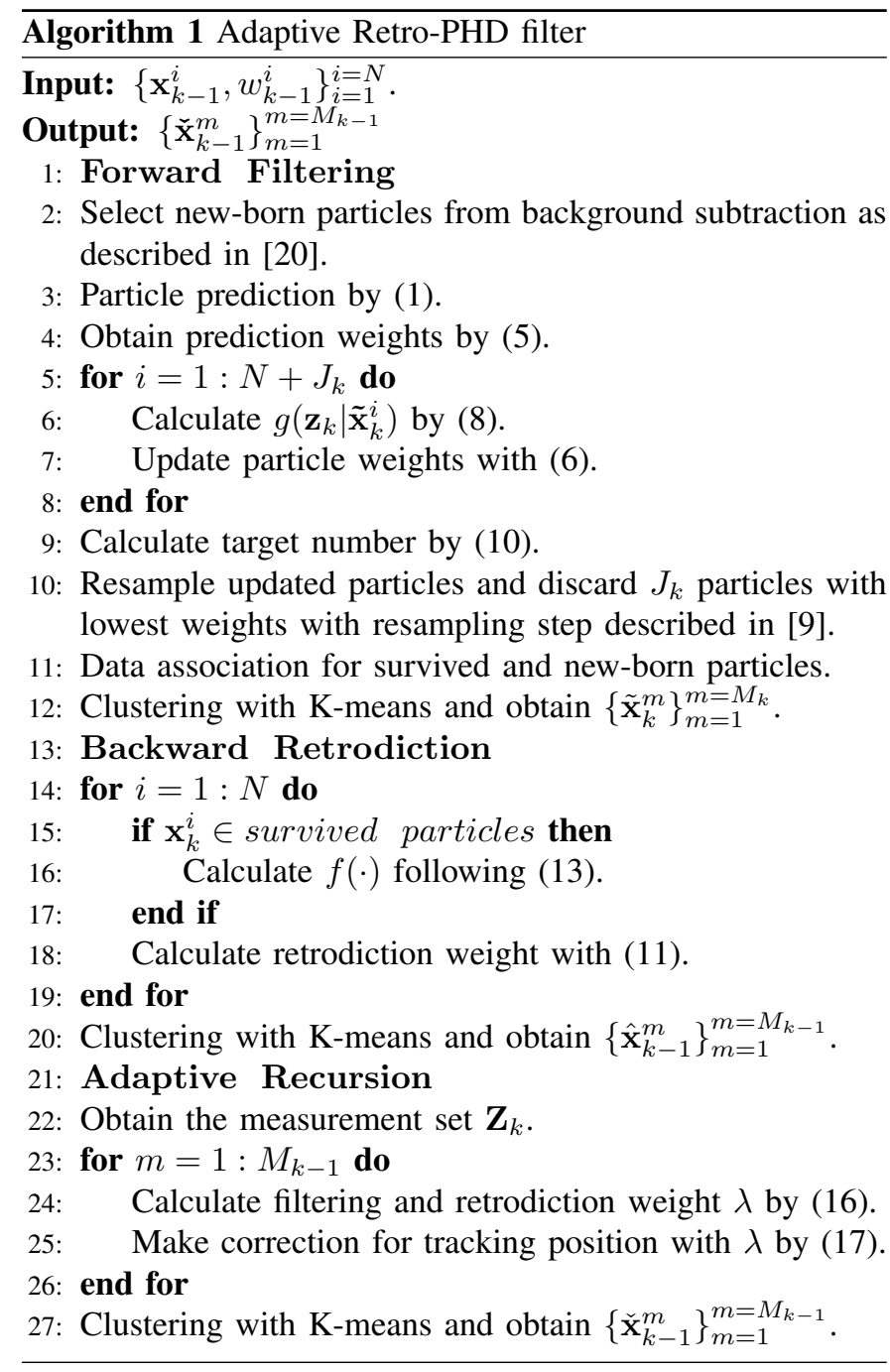

$\operatorname{cov}\left\{\mathbf{w}_{k}\right\}=\operatorname{diag}\{25,25,16,16,4,4\}$ and for $\mathbf{v}_{k}, \operatorname{cov}\left\{\mathbf{v}_{k}\right\}=$ $\operatorname{diag}\{25,25\}$. The missed detection probability $p_{M}=0.01$, the survival probability $e=0.99$, the new born intensity is given as $\Upsilon=0.1$ and clutter intensity $\kappa=0.01$. The variance for conversion function $\sigma_{f}^{2}$, likelihood function $\sigma_{g}^{2}$ and $\lambda$ function $\sigma_{\lambda}^{2}$ are set empirically to be 25, 36 and 25 respectively. In order to reduce the computational complexity, the time lag $L$ is set to be 1 .

TABLE I

MEE COMPARISONS OF DIFFERENT TRACKING RESULTS

\begin{tabular}{|l|l|l|l|l|l|l|}
\hline & \multicolumn{3}{|c|}{ CAVIAR } & \multicolumn{3}{c|}{ PETS2009 } \\
\cline { 2 - 7 } & PHD & R-PHD & A-PHD & PHD & R-PHD & A-PHD \\
\hline ME (pixel) & 34.85 & 28.43 & 25.71 & 43.08 & 36.49 & 34.95 \\
\hline Improvement (\%) & - & $18.42 \%$ & $26.23 \%$ & - & $15.30 \%$ & $18.87 \%$ \\
\hline
\end{tabular}

In order to evaluate the proposed tracking approach, the mean of the Euclidean error (ME) between tracking results and the ground truth is compared and shown in Table [1] where PHD denotes the results from the PHD filter (i.e. the forward filtering step in Algorithem 1), R-PHD denotes the RetroPHD (i.e. forward filtering + the backward retrodiction step in Algorithem 1) and A-PHD denotes our proposed adaptive Retro-PHD filtering algorithm (i.e. the whole Algorithm 1).

The optimal subpattern assignment (OSPA) metric [22], which is popularly used by researchers is also employed, where the errors from both localization and cardinality are considered to evaluate the tracking system. The comparison of OSPA for both datasets is shown in Fig. 2 .

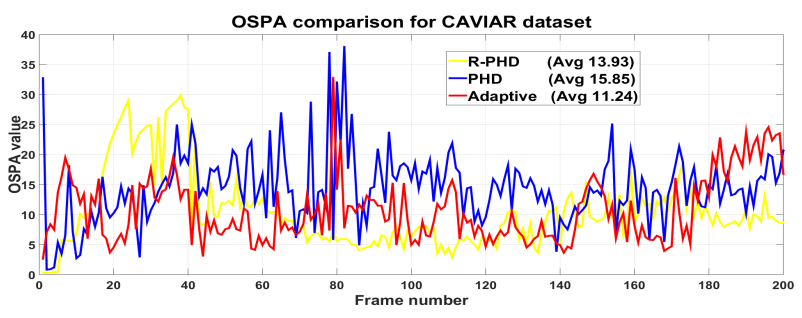

(a) OSPA comparison for CAVIAR dataset



(b) OSPA comparison for PETS2009 dataset

Fig. 2. Comparison of OSPA values between the PHD filtering (blue lines), Retro-PHD filtering (yellow lines) and proposed adaptive Retro-PHD filtering algorithms (red lines). Avg denotes the average value of OSPA from each tracking algorithm over the whole evaluation sequences.

We can find that for the CAVIAR dataset, the average value of OSPA is reduced by 1.92 and 4.61 when employing the Retro-PHD filtering and the proposed adaptive Retro-PHD filtering, respectively. For the PETS2009 dataset, the average value of OSPA is reduced by 0.03 and 2.48 respectively. The peak value around frame 80 of the CAVIAR dataset from the adaptive Retro-PHD is caused by the change of the number of targets and occlusion. From the comparison we can find, because of the false measurements caused by time-varying number of targets, missed detections and false alarms, the improvement from the Retro-PHD filter is limited in multiple human tracking. When employing the adaptive recursion step, however, the weights for the measurements from filtering and retrodiction are given based upon the observation extracted from the state. Because of this step, the tracking system becomes more accurate as verified by these experiments.

\section{CONCLUSION}

In this work, we use the term retrodiction PHD filtering in recognition of the approximation error in the PHD smoother, and propose an adaptive retrodiction step for the particle PHD filter with the aid of forward and backward measurements motivated by interacting adaptive filters. The results show the improvement by the proposed method over the conventional particle Retro-PHD filter both in terms of the localization and cardinality through the mean of the Euclidean error on each frame and OSPA measure, where the OSPA value is reduced by $29 \%$ for the CAVIAR dataset and $18 \%$ for the PETS2009 dataset. In future work, more extensive evaluation and comparisons will be performed. 


\section{REFERENCES}

[1] S. S. Blackman, "Multiple hypothesis tracking for multiple target tracking," IEEE Aerospace and Electronic Systems Magazine, vol. 19, no. 1 , pp. 5-18, 2004.

[2] E. Maggio and A. Cavallaro, Video Tracking. John Wiley and Sons, Ltd, 2011.

[3] E. Maggio, M. Taj, and A. Cavallaro, "Efficient multitarget visual tracking using random finite sets," IEEE Transactions on Circuits and Systems for Video Technology, vol. 18, no. 8, pp. 1016-1027, 2008.

[4] R. Mahler, "A theoretical foundation for the Stein-Winter Probability Hypothesis Density (PHD) multitarget tracking approach." DTIC Document, 2000.

[5] R. Mahler and L. Martin, "Multitarget Bayes filtering via first-order multitarget moments," IEEE Transactions on Aerospace and Electronic Systems., vol. 39, pp. 1152 - 1178, 2003.

[6] R. Mahler, "A theoretical foundation for the stein-winter "probability hypothesis density (PHD)" multitarget tracking approach," DTIC Document, Tech. Rep., 2000.

[7] O. Erdinc, P. Willett, and Y. Bar-Shalom, "Probability hypothesis density filter for multitarget multisensor tracking," in Proc. 8th International Conference on Information Fusion, vol. 1, pp. 146-153, 2005.

[8] N. Nadarajah, T. Kirubarajan, T. Lang, M. McDonald, and K. Punithakumar, "Multitarget tracking using probability hypothesis density smoothing," IEEE Transactions on Aerospace and Electronic Systems, vol. 47, no. 4, pp. 2344-2360, 2011.

[9] R. P. Mahler, B. T. Vo, and B. N. Vo, "Forward-backward probability hypothesis density smoothing," IEEE Transactions on Aerospace and Electronic Systems, vol. 48, no. 1, pp. 707-728, 2012.

[10] M. L. Psiaki, "Backward-smoothing extended Kalman filter," Journal of Guidance, Control and Dynamics, vol. 28, no. 5, pp. 885-894, 2005.

[11] J. Arenas-Garca, L. A. Azpicueta-Ruiz, M. T. M. Sliva, V. H. Nascimento, and A. H. Sayed, "Combinations of adaptive filters," IEEE Signal Processing Magzine, pp. 120-140, 2016.

[12] Y. Zhang and J. A. Chambers, "Convex combination of adaptive filters for a variable tap-length LMS algorithm," Signal Processing Letters, IEEE, vol. 13, no. 10 , pp. 628-631, 2006.

[13] R. Fisher. Caviar case scenarios. [Online]. Available: http://groups.inf. ed.ac.uk/vision/CAVIAR/CAVIARDATA1/

[14] I. Goldberg and M. J. Atallah. Privacy enhancing technologies, 9th international symposium, PETS2009. [Online]. Available: http: //dx.doi.org/10.1007/978-3-642-03168-7

[15] B. T. Vo, B. N. Vo and A. Cantoni, "The cardinalized probability hypothesis density filter for linear Gaussian multi-target models," in Proc. Annual Conference on Information Sciences and Systems, pp. 681686, 2006.

[16] R. Hoseinnezhad, B.N. Vo, and B.T. Vo, "Visual tracking in background subtracted image sequences via Multi-Bernoulli filtering," IEEE Transactions on Signal Processing, vol. 61, no. 2, pp. 392-397, 2013.

[17] B. N. Vo, S. Singh and A. Doucet, "Sequential Monte Carlo method for multi-target filtering with random finite sets," IEEE Transactions on Aerospace and Electronic Systems, vol. 41, no. 4, pp. 1224-1245, 2005.

[18] M. S. Arulampalam, S. Maskell, N. Gordon, and T. Clapp, "A tutorial on particle filters for online nonlinear/non-Gaussian Bayesian tracking." IEEE Transactions on Signal Processing, vol. 50, pp. 2454-2467, 2002.

[19] D. E. Clark and J. Bell, "Convergence results for the particle PHD filter," IEEE Transactions on Signal Processing, vol. 54, no. 7, pp. 2652-2661, 2006.

[20] P. Feng, W. Wang, S. Naqvi, S. Dlay, and J. Chambers, "Social force model aided robust particle PHD filter for multiple human tracking." in Proc. IEEE International Conference on Acoustics, Speech and Signal Processing., pp. 4398-4402, 2016.

[21] P. Feng, M. Yu, S. M. Naqvi, W. Wang and J. A. Chambers, "A robust Student's-t distribution PHD filter with OCSVM updating for multiple human tracking," in Proc. European Signal Processing Conference (EUSIPCO 2015), pp. 1-5, 2015.

[22] D. Schuhmacher, B. T. Vo, and B. N. Vo, "A consistent metric for performance evaluation of multi-object filters," IEEE Transactions on Signal Processing, vol. 56, no. 8, pp. 3447-3457, 2008. 